\title{
Diagnostic role of contrast-enhanced ultrasonography versus conventional B-mode ultrasonography in cirrhotic patients with early hepatocellular carcinoma: a retrospective study
}

\author{
Yandong Jiang, Meng Zhang, Yanting Zhu, Dongxiao Zhu \\ Department of Ultrasound, Affiliated Hospital of Jiangnan University, Wuxi, China \\ Contributions: (I) Conception and design: Y Jiang, D Zhu; (II) Administrative support: D Zhu; (III) Provision of study materials or patients: Y Jiang, \\ M Zhang, Y Zhu; (IV) Collection and assembly of data: Y Jiang, M Zhang, Y Zhu; (V) Data analysis and interpretation: Y Jiang; (VI) Manuscript \\ writing: All authors; (VII) Final approval of manuscript: All authors. \\ Correspondence to: Dongxiao Zhu. Department of Ultrasound, Affiliated Hospital of Jiangnan University, Wuxi 214122, China. \\ Email: zdx234567@126.com.
}

Background: Early diagnosis of hepatocellular carcinoma (HCC) is very important for the prognosis of patients. However, there are very few studies that compared the diagnostic accuracy of contrast-enhanced ultrasonography (CEUS) and B-mode ultrasonography for early HCC in cirrhotic patients.

Methods: This retrospective study included cirrhotic patients, who were suspected of early HCC between January 2020 and June 2021. The diagnosis of patients was based on the pathology results of surgery or biopsy. Demographic and clinical characteristics of included patients were recorded. The diagnoses of suspected lesions using both types of ultrasonography were recorded, and the sensitivity, specificity, positive predictive value (PPV), negative predictive value (NPV), and diagnostic accuracy of early HCC in cirrhotic patients were calculated.

Results: Eventually, 137 patients with solitary lesions in the liver were included in this study, including 89 patients diagnosed with HCC and 48 patients diagnosed with non-HCC. The median diameter of suspected lesions was $26 \mathrm{~mm}$, and the median level of alpha fetoprotein (AFP) was $37.2 \mathrm{ng} / \mathrm{mL}$. When comparing the demographic and clinical characteristics of cirrhotic patients with HCC and non-HCC, it was found that patients with HCC had significantly higher levels of AFP than those with non-HCC $(\mathrm{P}=0.03)$. The sensitivity, specificity, PPV, NPV, and accuracy of CEUS in early HCC were 73\%, 93.8\%, 95.6\%, $65.2 \%$ and $80.3 \%$, respectively. In CEUS, all of these parameters were much higher than those in B-mode ultrasonography, i.e., $64 \%, 75 \%, 82.6 \%, 52.9 \%$, and $67.9 \%$. It was also found that the diagnostic accuracy of CEUS was much higher than that of B-mode ultrasonography especially regarding lesions $<20 \mathrm{~mm}$. To further improve the sensitivity of CEUS in early HCC, AFP was combined with CEUS for the diagnosis of early HCC. As a result, the sensitivity, specificity, PPV, NPV, and accuracy of CEUS combined with AFP level were $83.1 \%, 87.5 \%, 92.5 \%, 73.7 \%$, and $84.7 \%$, respectively.

Conclusions: Our study confirmed that CEUS' diagnostic accuracy for early HCC in cirrhotic patients was significantly higher than that of B-mode ultrasonography. However, the sensitivity of CEUS needs to be improved further, and the combination of CEUS and AFP level may be a potential solution.

Keywords: Contrast-enhanced ultrasonography (CEUS); B-mode ultrasonography; alpha fetoprotein (AFP); diagnostic accuracy; early hepatocellular carcinoma (early HCC)

Submitted Aug 31, 2021. Accepted for publication Oct 14, 2021.

doi: 10.21037/jgo-21-611

View this article at: https://dx.doi.org/10.21037/jgo-21-611

(c) Journal of Gastrointestinal Oncology. All rights reserved. 


\section{Introduction}

Hepatocellular carcinoma (HCC) is one of the most common malignancies of the digestive tract, and liver cirrhosis, caused by viral hepatitis or alcoholic liver disease, is one of the most important risk factors for HCC (1-3). The incidence rate of HCC is high all over the world and has been increasing by $2 \%$ to $3 \%$ annually since 2007 (4). It has been reported that the cases of, and deaths from, HCC in China account for about half of cases worldwide (5). Surgical treatment is the most effective treatment for HCC, but it is limited to early-stage HCC. Local treatment can also alleviate the progress of the disease to a certain extent. However, once the invasion of surrounding organs and distant metastasis in HCC occurs in patients, drug treatment, including chemotherapy drugs, targeted drugs, and immune checkpoint inhibitors must be administered. Therefore, early diagnosis of HCC is very important for the prognosis of patients.

The advantages of B-mode ultrasonography are its low cost, short amount of time taken to complete the procedure, and high sensitivity to detect large tumors. Hence, it is the most commonly used abdominal examination tool. At present, all HCC guidelines also recommend B-mode ultrasonography as an initial screening tool for early HCC in patients with liver cirrhosis (6). Previous studies have demonstrated that B-ultrasonography has good specificity and overall accuracy in the diagnosis of liver tumors (7). However, the morphological structure and echo characteristics of cirrhotic livers are abnormal, and imaging does not always identify early HCC, which leads to low sensitivity of B-mode ultrasonography in the diagnosis of early HCC and a tendency to missed diagnoses (8). To make up for this shortcoming, contrast-enhanced computed tomography (CT) and magnetic resonance imaging (MRI) are often used for further examination of HCC after initial screening using B-mode ultrasonography. Previous studies have shown that contrast-enhanced CT and MRI have higher sensitivity and specificity in the diagnosis of early HCC compared with B-mode ultrasonography $(9,10)$. However, contrast-enhanced CT and MRI are expensive and time-consuming compared with B-mode ultrasonography. In addition, some patients may be allergic to the contrast agents used for contrast-enhanced CT and MRI. These factors limit the application of contrast-enhanced CT and MRI in the diagnosis of early HCC. Meanwhile, the advantages of contrast-enhanced ultrasonography (CEUS) mean that it is more widely used in the diagnosis of early
HCC. Also, CEUS uses intravenous injection of contrast agent and conventional ultrasonography, which can detect blood perfusion and microvascular distribution of organs and tumors and has almost no side-effects (11-13). It has been reported that CEUS acts as an effective examination tool for early HCC, and that the specificity of CEUS is higher than that of contrast-enhanced CT and MRI $(4,9)$. In a randomized controlled trial, Kudo et al. demonstrated that CEUS had significantly higher sensitivity and overall accuracy than B-mode ultrasonography in the diagnosis of early HCC (14). However, there were few patients with HCC in this study, which limits the clinical applicability of the results. However, there are no other similar studies, which compared the diagnostic accuracy of CEUS and B-mode ultrasonography in the diagnosis of HCC.

Our study was performed to compare the diagnostic role of CEUS and B-mode ultrasonography in early HCC by retrospectively collecting the characteristics and diagnoses of cirrhotic patients admitted to our hospital during a 1.5 -year period. To remove the heterogeneity between included patients, we only included cirrhotic patients receiving both CEUS and B-mode ultrasonography. The aim of our study was to further verify the superiority of CEUS compared with B-mode ultrasonography in the diagnosis of early HCC. We present the following article in accordance with the STARD reporting checklist (available at https://dx.doi.org/10.21037/jgo-21-611).

\section{Methods}

\section{Patient recruitment}

Our study was approved by the ethics committee of the Affiliated Hospital of Jiangnan University (No. 2021-066) and then performed according to the principles of the Helsinki Declaration [2013]. Cirrhotic patients who were suspected of early HCC between January 2020 and June 2021 were included in this study. The diagnosis of patients was based on the pathological results (the gold standard) once surgery or biopsy had been performed. Patients with the following patient characteristics were excluded from this study: younger than 18 years old, did not have liver cirrhosis, received only one examination of CEUS and B-mode ultrasonography, did not undergo surgery or biopsy for definite diagnosis, was eventually diagnosed as having multiple lesions of HCC or a solitary lesion of HCC with a diameter exceeding $5 \mathrm{~cm}$, and could not provide their comprehensive characteristics. Due to the retrospective 
design, written informed consents were not obtained in this study. All included patients were followed up at least until pathological results were obtained. Some demographic and clinical characteristics of included patients were extracted from their medical records, including age, gender, body mass index (BMI), etiology of cirrhosis [hepatitis $\mathrm{B}$ virus (HBV) related cirrhosis, hepatitis $\mathrm{C}$ virus (HCV) related cirrhosis, alcohol-related cirrhosis or other cirrhosis], Child-Pugh classification (A, B or C), lesion location (major branch or segmental branch), lesion size, and level of alpha fetoprotein (AFP).

\section{$B$-mode ultrasonography and CEUS}

Both B-mode ultrasonography and CEUS were performed by skilled radiologists using a medical ultrasound system (EPIQ 5, Bothell, WA, USA) with a 1-5 MHz convex transducer. First, B-mode ultrasonography was performed using gray-scale ultrasound. According to the suspected lesions detected by B-mode ultrasonography, CEUS was performed using contrast-enhanced imaging after the injection of 1-2 $\mathrm{mL}$ contrast medium of sulfur hexafluoride (SonoVue, Bracco, Italy) into the location of the lesion followed by $5-10 \mathrm{~mL}$ of normal saline. The contrast medium was administered a second time if necessary. The suspected lesion and nearby liver parenchyma were detected for about one minute, then whole liver parenchyma and the suspected lesion were detected until washout took place. The diagnosis of suspected lesions in this study was based on the judgement of one to three skilled radiologists, who did not have access to demographic and clinical characteristics of included patients.

\section{Statistical analysis}

Statistical analysis in this study was performed using SPSS 18.0 (IBM Corp., New York City, NY, USA). Continuous variables are reported as medians and quartiles and compared using Mann-Whitney U tests. Categorical variables are reported as frequencies and percentages and compared using chi square tests. A two-tailed $\mathrm{P}$ value $<0.05$ was considered as indicating a statistically significant difference. The diagnoses of suspected lesions using both examinations were recorded, and the sensitivity, specificity, positive predictive value (PPV), negative predictive value (NPV), and accuracy of both examinations of early HCC in cirrhotic patients were calculated. Also, the diagnostic role of the AFP level in early HCC was measured, and then the diagnostic role of CEUS was combined with the AFP level measured to determine whether AFP level could further improve the sensitivity and overall accuracy of CEUS in the diagnosis of early HCC in cirrhotic patients.

\section{Results}

More than 30,000 patients were admitted to our hospital for abdominal B-mode ultrasonography between January 2020 and June 2021, of whom 469 patients also received examination of CEUS. In all, 332 patients were excluded from this study according to the exclusion criteria. Eventually, 137 patients with solitary lesions in the liver were included in this study (Figure 1). According to the pathological results of surgery or biopsy, 89 patients were diagnosed as HCC, and 48 patients were diagnosed as nonHCC.

Demographic and clinical characteristics of cirrhotic patients are shown in Table 1. Median age was 56 years, 99 patients $(72.3 \%)$ were male, and the median BMI was 23.1. In about three quarters of included patients the cirrhosis was caused by HBV. More than $90 \%$ patients were diagnosed as Child-Pugh classification A. Also, about three quarters of suspected lesions were located at a major branch. The median diameter of suspected lesions was $26 \mathrm{~mm}$, and the median level of AFP was $37.2 \mathrm{ng} / \mathrm{mL}$. Eighty-nine lesions $(65.0 \%)$ were eventually diagnosed as HCC (Table 2). Nineteen of the remaining lesions were non-HCC malignancies, including 12 intrahepatic cholangiocarcinomas (ICCs), one HCC combined with ICC, and six metastatic lesions. Twenty-nine lesions were benign, including 12 dysplastic nodules, 10 regenerative nodules, three hemangioma, two focal nodular hyperplasias, and two inflammatory pseudotumors. Comparing demographic and clinical characteristics of cirrhotic patients with HCC or non-HCC in this study, it was found that patients with HCC had significantly higher levels of AFP than those with non-HCC patients (Table 3). No additional differences were found between them.

Two representative patients with early HCC of diameters of $20 \mathrm{~mm}$ are shown in Figure 2. It was found that a small $\mathrm{HCC}$ was hypoechoic in B-mode ultrasonography, but that it was not easy to distinguish it from other lesions. In addition, a small HCC in CEUS showed hyperenhancement in the arterial phase and hypoenhancement in the portal and delayed phase, which can be distinguished from other lesions. The diagnostic roles of CEUS and B-mode ultrasonography are listed in Table 4. The sensitivity, 


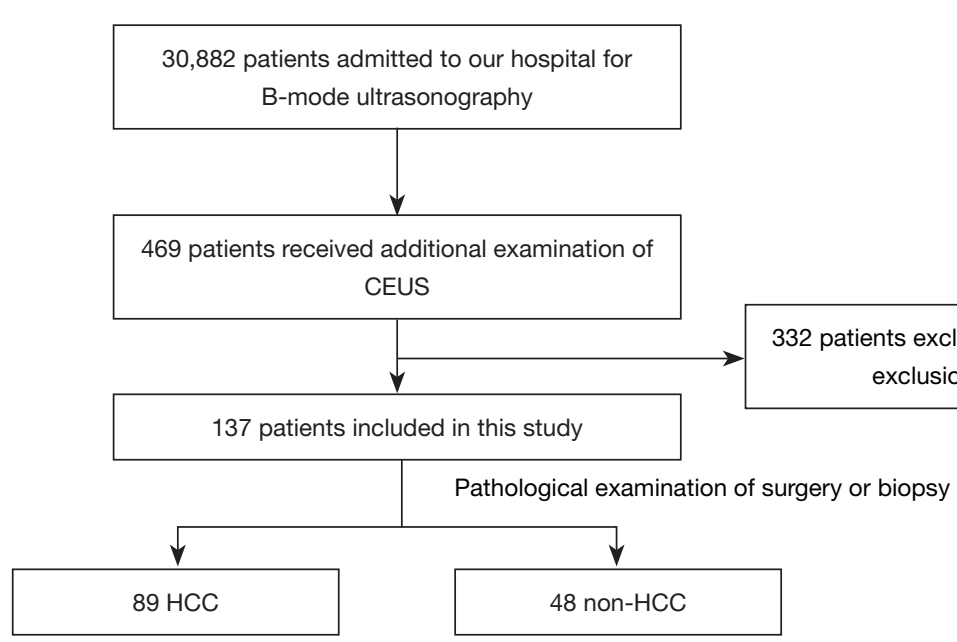

Figure 1 Flow chart. CEUS, contrast-enhanced ultrasonography; HCC, hepatocellular carcinoma.

Table 1 Demographic and clinical characteristics of cirrhotic patients

\begin{tabular}{|c|c|}
\hline Characteristics & Data \\
\hline Number & 137 \\
\hline Age, year & $56(49.0-63.0)$ \\
\hline \multicolumn{2}{|l|}{ Gender } \\
\hline Male & 99 (72.3\%) \\
\hline Female & $38(27.7 \%)$ \\
\hline BMI & $23.1(21.6-24.5)$ \\
\hline \multicolumn{2}{|l|}{ Etiology of cirrhosis } \\
\hline HBV related & $101(73.7 \%)$ \\
\hline $\mathrm{HCV}$ related & $8(5.8 \%)$ \\
\hline Alcohol related & $22(16.1 \%)$ \\
\hline Other & $6(4.4 \%)$ \\
\hline \multicolumn{2}{|c|}{ Child-Pugh classification } \\
\hline A & 125 (91.2\%) \\
\hline B & $12(8.8 \%)$ \\
\hline \multicolumn{2}{|l|}{ Lesion location } \\
\hline Major branch & 100 (73.0\%) \\
\hline Segmental branch & $37(27.0 \%)$ \\
\hline Lesion size, mm & $26(18.0-42.0)$ \\
\hline Level of AFP, ng/mL & $37.2(8.6-655)$ \\
\hline
\end{tabular}

Table 2 Diagnosis of liver lesion

\begin{tabular}{lc}
\hline Final diagnosis & Data \\
\hline HCC & $89(65.0 \%)$ \\
Non-HCC malignancy & $19(13.9 \%)$ \\
ICC & $12(8.8 \%)$ \\
Combined HCC and ICC & $1(0.7 \%)$ \\
Metastasis & $6(4.4 \%)$ \\
Benign lesions & $29(21.2 \%)$ \\
Dysplastic nodule & $12(8.8 \%)$ \\
Regenerative nodule & $10(7.3 \%)$ \\
Hemangioma & $3(2.2 \%)$ \\
Focal nodular hyperplasia & $2(1.5 \%)$ \\
Inflammatory pseudotumor & $2(1.5 \%)$ \\
\hline HCC, hepatocellular carcinoma; ICC, intrahepatic cholangiocarcinoma.
\end{tabular}

specificity, PPV, NPV, and accuracy of CEUS in early HCC were $73 \%, 93.8 \%, 95.6 \%, 65.2 \%$, and $80.3 \%$, respectively. All of these parameters in CEUS were much higher than those in B-mode ultrasonography, i.e., $64 \%, 75 \%, 82.6 \%$, $52.9 \%$, and $67.9 \%$. Then, these lesions were divided into large lesions $(\geq 20 \mathrm{~mm})$ and small lesions $(<20 \mathrm{~mm})$, and diagnostic roles of CEUS and B-mode ultrasonography in suspected HCCs with different diameters were determined (Table 5). It was found that diagnostic accuracy of CEUS was much higher than that of B-mode ultrasonography 
Table 3 Comparisons of demographic and clinical characteristics of cirrhotic patients with hepatocellular carcinoma (HCC) or non-HCC

\begin{tabular}{|c|c|c|c|}
\hline Characteristics & $\mathrm{HCC}$ & Non-HCC & $P$ value \\
\hline Age, year & $59(46.0-65.0)$ & $57(49.0-64.0)$ & 0.98 \\
\hline Gender & & & 0.60 \\
\hline Male & $63(70.8 \%)$ & $36(75.0 \%)$ & \\
\hline BMI & $23.1(21.4-24.5)$ & $22.9(21.6-24.1)$ & 0.55 \\
\hline Etiology of cirrhosis & & & 0.15 \\
\hline HBV related & $60(67.4 \%)$ & $41(85.4 \%)$ & \\
\hline HCV related & $6(6.7 \%)$ & $2(4.2 \%)$ & \\
\hline Child-Pugh classification & & & 0.61 \\
\hline$A$ & $82(92.1 \%)$ & $43(89.6 \%)$ & \\
\hline B & $7(7.9 \%)$ & $5(10.4 \%)$ & \\
\hline Lesion location & & & 0.11 \\
\hline Major branch & $61(68.5 \%)$ & $39(81.3 \%)$ & \\
\hline Segmental branch & $28(31.5 \%)$ & $9(18.7 \%)$ & \\
\hline Lesion size, mm & $26(20.0-42.0)$ & $26(19.0-39.0)$ & 0.83 \\
\hline Level of AFP, ng/mL & $58.0(14.9-1,366)$ & $22.5(9.3-219)$ & 0.03 \\
\hline
\end{tabular}

HCC, hepatocellular carcinoma; ICC, intrahepatic cholangiocarcinoma; BMI, body mass index; AFP, alpha fetoprotein; HBV, hepatitis B virus; HCV, hepatitis B virus.

especially in lesions $<20 \mathrm{~mm}$. To further improve the sensitivity of CEUS in early HCC, AFP was chosen as an auxiliary diagnostic tool of CEUS, because in this study the levels of AFP in HCC were much higher than those in non-HCC. As a result, the sensitivity, specificity, PPV, NPV, and accuracy of CEUS combined with AFP levels achieved were up to $83.1 \%, 87.5 \%, 92.5 \%, 73.7 \%$, and $84.7 \%$, respectively (Table 6). This significantly improved the sensitivity of CEUS in early HCC without reducing the specificity of CEUS too significantly.

\section{Discussion}

Our study included more cirrhotic patients with HCC than previous studies and demonstrated higher sensitivity of CEUS compared to B-mode ultrasonography in the diagnosis of early HCC. The results showed that with the use of contrast medium, CEUS assessed the vascular distribution and blood supply of liver lesions more effectively and thus diagnosed HCC more accurately than B-mode ultrasonography, regardless of whether tumors had a diameter greater or less than $2 \mathrm{~cm}$. However, in general, the sensitivity of CEUS in the diagnosis of HCC was still not particularly high. Therefore, this study attempted to combine AFP levels and CEUS in the diagnosis of early HCC. The results showed that combined AFP levels and CEUS had higher sensitivity and diagnostic accuracy than AFP level or CEUS alone. The results of this study demonstrate that there are several options for the diagnosis of early HCC.

B-mode ultrasonography is the most commonly used abdominal examination tool, and it is mainly used in hepatobiliary pancreatic diseases and gynecological diseases. However, in recent years few studies have focused on the diagnosis of cirrhotic patients with early HCC using B-mode ultrasonography. Previous studies have pointed out that the 

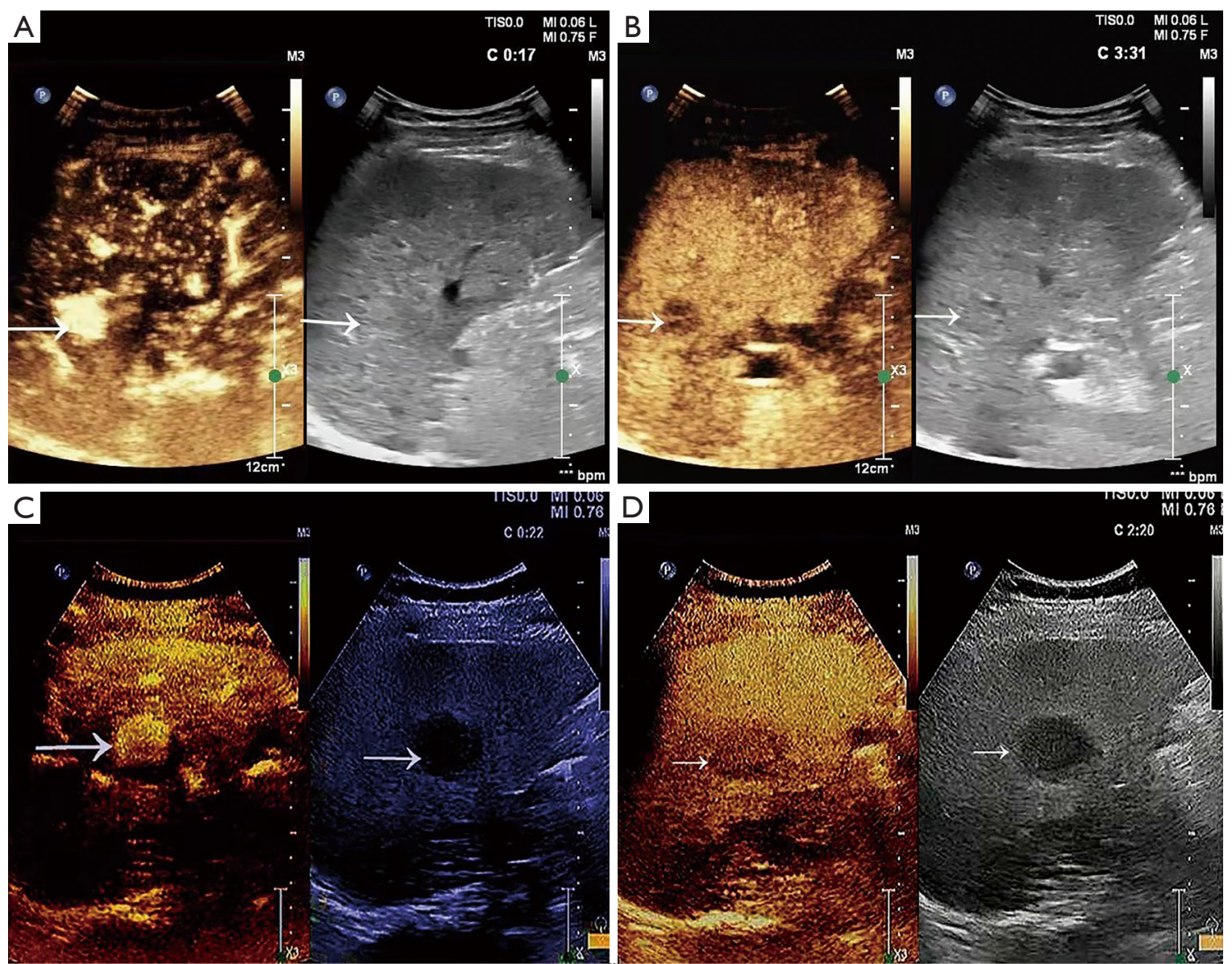

Figure 2 Representative imaging of two cirrhotic patients with early similar HCC using CEUS (left) and B-mode ultrasonography (right). Patient 1 with early HCC with a diameter of $16 \mathrm{~mm} \times 15 \mathrm{~mm}$ (A: arterial phase, B: portal and delayed phases) and Patient 2 with early HCC with a diameter of $18 \mathrm{~mm} \times 16 \mathrm{~mm}$ (C: arterial phase, D: portal and delayed phases). Early HCC in both patients showing hyperenhancement in the arterial phase and hypoenhancement in the portal and delayed phase. The arrows refer to the location of HCC. HCC, hepatocellular carcinoma; CEUS, contrast-enhanced ultrasonography.

Table 4 Diagnostic roles of contrast-enhanced ultrasonography and B-mode ultrasonography in cirrhotic patients suspected of hepatocellular carcinoma

\begin{tabular}{lcc}
\hline Variable & CEUS & $\begin{array}{c}\text { B-mode } \\
\text { ultrasonography }\end{array}$ \\
\hline Sensitivity & 73 & 64 \\
Specificity & 93.8 & 75 \\
PPV & 95.6 & 82.6 \\
NPV & 65.2 & 52.9 \\
Accuracy & 80.3 & 67.9 \\
\hline
\end{tabular}

PPV, positive predictive value; NPV, negative predictive value; CEUS, contrast-enhanced ultrasonography. sensitivity and specificity of B-mode ultrasonography in the diagnosis of HCC are $50-60 \%$ and $70-80 \%$, respectively (14-16), which is similar to our results. It should also be noted that the overall accuracy in the diagnosis of early HCC was relatively low. Therefore, further assessments are needed to diagnose suspected HCC after ultrasonography, such as CT and MRI. However, the two examinations are expensive and time-consuming, which can easily lead to the delay of the disease. Contrast-enhanced ultrasonography is an advanced B-ultrasonography examination tool. In China, the contrast agent used is SonoVue, a type of sulfur hexafluoride, which rarely leads to serious allergic reactions and adverse reactions and allows clearer imaging and more 
Table 5 Diagnostic roles of contrast-enhanced ultrasonography and B-mode ultrasonography in cirrhotic patients suspected of hepatocellular carcinoma with different diameters

\begin{tabular}{lcccc}
\hline Variable & $\begin{array}{c}\text { CEUS-lesion } \\
<20 \mathrm{~mm}\end{array}$ & $\begin{array}{c}\text { B-mode ultrasonography-lesion } \\
<20 \mathrm{~mm}\end{array}$ & $\begin{array}{c}\text { CEUS-lesion } \\
\geq 20 \mathrm{~mm}\end{array}$ & $\begin{array}{c}\text { B-mode ultrasonography-lesion } \\
\geq 20 \mathrm{~mm}\end{array}$ \\
\hline Sensitivity & 69 & 51.7 & 75 & 70 \\
Specificity & 86.7 & 66.7 & 97 & 78.8 \\
PPV & 90.9 & 75 & 97.8 & 85.7 \\
NPV & 59.1 & 41.2 & 68.1 & 59.1 \\
Accuracy & 75 & 56.8 & 82.8 & 73.1 \\
\hline
\end{tabular}

PPV, positive predictive value; NPV, negative predictive value; CEUS, contrast-enhanced ultrasonography.

Table 6 Diagnostic role of contrast-enhanced ultrasonography combined with alpha fetoprotein level in cirrhotic patients suspected of hepatocellular carcinoma

\begin{tabular}{lc}
\hline Variable & CEUS combined with AFP level \\
\hline Sensitivity & 83.1 \\
Specificity & 87.5 \\
PPV & 92.5 \\
NPV & 73.7 \\
Accuracy & 84.7 \\
\hline
\end{tabular}

PPV, positive predictive value; NPV, negative predictive value; CEUS, contrast-enhanced ultrasonography; AFP, alpha fetoprotein.

accurate diagnosis (17-19). Previous studies mostly showed that CEUS achieved about 70-90\% sensitivity and about $80-100 \%$ specificity in the diagnosis of HCC $(14,20-22)$. In our study, the sensitivity of CEUS in the diagnosis of early liver cancer was $73.0 \%$, while the sensitivity was $93.8 \%$, which was significantly superior to the $64.0 \%$ sensitivity and $75.0 \%$ specificity of B-mode ultrasonography. This provides a theoretical basis for CEUS to replace B-mode ultrasonography as a novel screening tool of early HCC, but the premise is to further reduce the cost of the contrast medium.

We also found that the sensitivity of CEUS in the diagnosis of early HCC in cirrhotic patients was still not high. Therefore, we also sought to identify an auxiliary examination tool to improve the sensitivity of CEUS in early HCC diagnosis. AFP is one of the most commonly used serum markers for the diagnosis of HCC. In our study, most patients underwent measurement of AFP level after identifying a liver lesion. AFP was then chosen as the auxiliary diagnostic tool of CEUS, and CEUS was combined with AFP level to diagnose early HCC in cirrhotic patients. When selecting the diagnostic cut-off point of AFP, we referred to previous studies. A higher cutoff point will lead to higher specificity and lower sensitivity, while a lower cut-off point will slightly reduce the specificity and significantly improve the sensitivity (23-25). We therefore chose the lower AFP cut-off point, $10 \mathrm{ng} / \mathrm{mL}$, and early HCC was diagnosed when the patient met any of the diagnostic criteria of CEUS or AFP level. This led to the sensitivity of combined diagnostic criteria achieving $83.1 \%$ with the specificity dropping to $87.5 \%$, which meant that the specificity and sensitivity reached a relatively satisfactory level.

This study has some limitations. First, the patients included in this study were first screened using B-mode ultrasonography. Only those patients who were confirmed using B-mode ultrasonography to have a solitary liver lesion were further examined using CEUS. Tumors too small in diameter or too difficult to distinguish on B-mode ultrasonography were not included in the study. This may have led to a certain degree of selection bias in this study, which was mainly due to the retrospective design of the study, a shortcoming that needs to be addressed in future prospective studies. Second, the patients included in this study were all patients with liver cirrhosis, but there were some differences in the etiology of liver cirrhosis, including cirrhosis caused by HBV, HCV, and alcohol, which may have led to the difference in the phenotypes of HCC and the surrounding liver parenchyma. When the sample size is adequate, the diagnosis of HCC in patients with liver cirrhosis caused by a single cause may be able to demonstrate the accuracy of CEUS and B-ultrasonography. Third, this study was a retrospective study, und thus the 
selection of serum markers was relatively limited, and only the more commonly used serum markers in the clinical setting were selected for analysis. AFP is the most commonly used serum marker for suspected HCC in China, but other new markers may have higher diagnostic accuracy. More in-depth future research should be carried out.

\section{Conclusions}

Few studies on the accuracy of CEUS and B-mode ultrasonography in the diagnosis of HCC are available. This study included cirrhotic patients suspected of HCC, and the results showed that CEUS had a higher diagnostic accuracy than B-mode ultrasonography for HCCs with both large and small diameters. However, the sensitivity of CEUS in the diagnosis of HCC was still low. This study attempted to combine AFP levels and CEUS in the diagnosis of HCC. It was found that the sensitivity and accuracy of the combined diagnostic criteria for HCC were significantly improved. Contrast-enhanced ultrasonography and AFP are commonly used imaging examination tools and serum markers, respectively, and the combination of the two has significant clinical significance and provides a new approach to the diagnosis of early HCC.

\section{Acknowledgments}

Funding: None.

\section{Footnote}

Reporting Checklist: The authors have completed the STARD reporting checklist. Available at https://dx.doi. org/10.21037/jgo-21-611

Data Sharing Statement: Available at https://dx.doi. org/10.21037/jgo-21-611

Conflicts of Interest: All authors have completed the ICMJE uniform disclosure form (available at https://dx.doi. org/10.21037/jgo-21-611). The authors have no conflicts of interest to declare.

Ethical Statement: The authors are accountable for all aspects of the work in ensuring that questions related to the accuracy or integrity of any part of the work are appropriately investigated and resolved. Our study was approved by the ethics committee of the Affiliated Hospital of Jiangnan University (No. 2021-066) and then performed according to the principles of the Helsinki Declaration [2013]. Due to the retrospective design, written informed consents were not obtained in this study.

Open Access Statement: This is an Open Access article distributed in accordance with the Creative Commons Attribution-NonCommercial-NoDerivs 4.0 International License (CC BY-NC-ND 4.0), which permits the noncommercial replication and distribution of the article with the strict proviso that no changes or edits are made and the original work is properly cited (including links to both the formal publication through the relevant DOI and the license). See: https://creativecommons.org/licenses/by-nc-nd/4.0/.

\section{References}

1. Zhang HC, Zhu T, Hu RF, et al. Contrast-enhanced ultrasound imaging features and clinical characteristics of combined hepatocellular cholangiocarcinoma: comparison with hepatocellular carcinoma and cholangiocarcinoma. Ultrasonography 2020;39:356-66.

2. Yang J, Zhang YH, Li JW, et al. Contrast-enhanced ultrasound in association with serum biomarkers for differentiating combined hepatocellularcholangiocarcinoma from hepatocellular carcinoma and intrahepatic cholangiocarcinoma. World J Gastroenterol 2020;26:7325-37.

3. Wu S, Lin X. Trials in developing a nanoscale material for extravascular contrast-enhanced ultrasound targeting hepatocellular carcinoma. PeerJ 2020;8:e10403.

4. Li S, Zhou L, Chen R, et al. Diagnostic efficacy of contrast-enhanced ultrasound versus MRI Liver Imaging Reporting and Data System (LI-RADS) for categorising hepatic observations in patients at risk of hepatocellular carcinoma. Clin Radiol 2021;76:161.e1-161.e10.

5. Zhang J, Yu Y, Li Y, et al. Diagnostic value of contrastenhanced ultrasound in hepatocellular carcinoma: a metaanalysis with evidence from 1998 to 2016. Oncotarget 2017;8:75418-26.

6. Dong Y, Wang WP, Mao F, et al. Imaging Features of Fibrolamellar Hepatocellular Carcinoma with ContrastEnhanced Ultrasound. Ultraschall Med 2021;42:306-13.

7. Mitrea D, Badea R, Mitrea P, et al. Hepatocellular Carcinoma Automatic Diagnosis within CEUS and B-Mode Ultrasound Images Using Advanced Machine Learning Methods. Sensors (Basel) 2021;21:2202.

8. Gregory J, Paisant A, Paulatto L, et al. Limited added 
value of contrast-enhanced ultrasound over B-mode for the subtyping of hepatocellular adenomas. Eur J Radiol 2020;128:109027.

9. Schwarze V, Marschner C, Völckers W, et al. Diagnostic value of contrast-enhanced ultrasound versus computed tomography for hepatocellular carcinoma: a retrospective, single-center evaluation of 234 patients. J Int Med Res 2020;48:300060520930151.

10. Tselikas L, Pigneur F, Roux M, et al. Impact of hepatobiliary phase liver MRI versus Contrast-Enhanced Ultrasound after an inconclusive extracellular gadolinium-based contrastenhanced MRI for the diagnosis of benign hepatocellular tumors. Abdom Radiol (NY) 2017;42:825-32.

11. Kang HJ, Lee JM, Yoon JH, et al. Role of ContrastEnhanced Ultrasound as a Second-Line Diagnostic Modality in Noninvasive Diagnostic Algorithms for Hepatocellular Carcinoma. Korean J Radiol 2021;22:354-65.

12. Schellhaas B, Bernatik T, Bohle W, et al. ContrastEnhanced Ultrasound Algorithms (CEUS-LIRADS/ ESCULAP) for the Noninvasive Diagnosis of Hepatocellular Carcinoma - A Prospective Multicenter DEGUM Study. Ultraschall Med 2021;42:178-86.

13. Wang F, Numata K, Okada M, et al. Comparison of Sonazoid contrast-enhanced ultrasound and gadoliniumethoxybenzyl-diethylenetriamine pentaacetic acid MRI for the histological diagnosis of hepatocellular carcinoma. Quant Imaging Med Surg 2021;11:2521-40.

14. Kudo M, Ueshima K, Osaki Y, et al. B-Mode Ultrasonography versus Contrast-Enhanced Ultrasonography for Surveillance of Hepatocellular Carcinoma: A Prospective Multicenter Randomized Controlled Trial. Liver Cancer 2019;8:271-80.

15. Moribata K, Tamai H, Shingaki N, et al. Assessment of malignant potential of small hypervascular hepatocellular carcinoma using B-mode ultrasonography. Hepatol Res 2011;41:233-9.

16. Nakagawara H, Ogawa M, Matsumoto N, et al. Evaluation of malignancy of hepatocellular carcinoma using the ultrasonic B-mode method: clinical significance of extracapsular invasion of hepatocellular carcinoma using ultrasonography. J Med Ultrason (2001) 2007;34:83-91.

17. Ye J, Huang G, Zhang X, et al. Three-dimensional contrast-enhanced ultrasound fusion imaging predicts local tumor progression by evaluating ablative margin of radiofrequency ablation for hepatocellular carcinoma: a preliminary report. Int J Hyperthermia 2019;36:55-64.

18. Wang Y, Liao J, Qi W, et al. Predictive Value of
Conventional Ultrasound and Contrast-Enhanced Ultrasound in Early Recurrence of Hepatocellular Carcinoma after Surgical Resection. Ultrasound Med Biol 2016;42:1042-8.

19. Lu Q, Zhang XL, Han H, et al. Value of Perfusion Parameters for Differentiating Hepatocellular Carcinoma and Liver Metastasis With Hypervascularity and a Normal Hepatic Background on Contrast-Enhanced Ultrasound Imaging. J Ultrasound Med 2019;38:2601-8.

20. Wu W, Chen M, Yan K, et al. Evaluation of contrastenhanced ultrasound for diagnosis of dysplastic nodules with a focus of hepatocellular carcinoma in liver cirrhosis patients. Chin J Cancer Res 2015;27:83-9.

21. Schaible J, Stroszczynski C, Beyer LP, et al. Quantitative perfusion analysis of hepatocellular carcinoma using dynamic contrast enhanced ultrasound (CEUS) to determine tumor microvascularization. Clin Hemorheol Microcirc 2019;73:95-104.

22. Marschner CA, Zhang L, Schwarze V, et al. The diagnostic value of contrast-enhanced ultrasound (CEUS) for assessing hepatocellular carcinoma compared to histopathology; a retrospective single-center analysis of 119 patients1. Clin Hemorheol Microcirc 2020;76:453-8.

23. Lahmidani N, Hamdoun FZ, Lahlali M, et al. Prognostic Impact of Alpha Fetoprotein at Diagnosis on Overall Survival of Single Small Hepatocellular Carcinomas. Gulf J Oncolog 2020;1:64-7.

24. Nörthen A, Asendorf T, Walson PD, et al. Diagnostic value of alpha-1-fetoprotein (AFP) as a biomarker for hepatocellular carcinoma recurrence after liver transplantation. Clin Biochem 2018;52:20-5.

25. Yao M, Zhao J, Lu F. Alpha-fetoprotein still is a valuable diagnostic and prognosis predicting biomarker in hepatitis $B$ virus infection-related hepatocellular carcinoma. Oncotarget 2016;7:3702-8.

(English Language Editor: B. Meiser)

Cite this article as: Jiang Y, Zhang M, Zhu Y, Zhu D. Diagnostic role of contrast-enhanced ultrasonography versus conventional B-mode ultrasonography in cirrhotic patients with early hepatocellular carcinoma: a retrospective study. J Gastrointest Oncol 2021;12(5):2403-2411. doi: 10.21037/jgo-21611 